\title{
Progressive Multifocal Leukoencephalopathy and Rituximab: Time to Better Stratify the Risk?
}

\author{
Immovilli Paolo ${ }^{\mathrm{a}, \mathrm{d}}$, Rota Eugenia ${ }^{\mathrm{a}}$, Morelli Nicola ${ }^{\mathrm{a}}$, De Mitri Paola ${ }^{\mathrm{a}}$, Terlizzi Emilio ${ }^{\mathrm{a}}$, \\ Magnifico Fabiola ${ }^{a}$, Cavanna Luigi ${ }^{b}$, Parmeggiani Maria ${ }^{c}$, Guidetti Donata ${ }^{a}$
}

\begin{abstract}
Progressive multifocal leukoencephalopathy (PML) is a demyelinating disease emerging as a complication of some novel monoclonal antibody therapies. Disease risk and severity show a wide variability, with fatality ranging from $30 \%$ to $90 \%$, probably depending on many risk determinants as demonstrated for natalizumab-associated PML. Rituximab therapy is used to treat hematological malignancies and autoimmune disease, but little is known about PML risk stratification and management. We describe a clinical case with very early diagnosis, extensive treatment, but a fatal outcome, highlighting the need for future studies aimed to better understand PML risk.
\end{abstract}

Keywords: Progressive multifocal leukoencephalopathy; Rituximab; Risk stratification; Treatment; Diagnosis

\section{Introduction}

Progressive multifocal leukoencephalopathy (PML) is a demyelinating disease of the brain caused by the John Cunningham virus (JCV). PML was originally recognized as a rare complication of either hematological malignancies or systemic inflammatory disorders; however, a dramatic increase in the incidence of this condition occurred with the HIV epidemic. Moreover, the use of some novel monoclonal antibodies (such as rituximab, natalizumab, and efalizumab) therapies has been associated with an increase in the incidence of PML [1].

Rituximab is an anti-CD20 monoclonal antibody cur-

Manuscript accepted for publication October 15, 2013

${ }^{a}$ Neurology Unit, Guglielmo da Saliceto Hospital, Piacenza, Italy

${ }^{\mathrm{b}}$ Oncology Unit, Guglielmo da Saliceto Hospital, Piacenza, Italy

${ }^{\mathrm{c}}$ Microbiology Unit, Santa Maria Nuova Hospital, Reggio Emilia, Italy

${ }^{\mathrm{d}}$ Corresponding author: Immovilli Paolo, Via Cantone del Cristo n 2,

29121 Piacenza, Italy. Email: paolo.immovilli.md@gmail.com

doi: http://dx.doi.org/10.14740/jnr235w rently employed for treatment of patients with non-Hodgkin lymphoma (NHL) and several autoimmune diseases. It has been reported that inclusion of rituximab into standard chemotherapy regimes for NHL was associated with a significant higher incidence of PML (rate difference, 2.2/1,000 patient-years) [2], furthermore rituximab-related PML has a high mortality rate $(90 \%)$ [3].

Several studies have assessed the use of various drugs for the PML treatment but with conflicting results, showing in vitro efficacy against JCV but without a survival benefit.

We report a clinical case of rituximab-related PML, with early diagnosis, extensive treatment but a fatal outcome despite such management.

\section{Case Report}

A 75-year-old man was admitted to the Neurology Unit owing to difficulties in speaking and playing cards during the last 4 days.

He was affected by hypertension and a third degree atrium-ventricular block, which required a pacemaker implantation.

In 2009, a spleen biopsy revealed a large B cell lymphoma, stage 3A; since July 2009, he was treated with 6 CHOP (cyclophosphamide, hydroxydaunomycin, oncovicin, and prednisone), 8 rituximab cycles ( $375 \mathrm{mg} / \mathrm{m}^{2}$ every 21 days), and spleen radiotherapy without any relapse; in November 2010 , a positron emission tomography scan was negative.

In February 2011, he complained of speaking and playing cards difficulties. The neurological examination was normal except for very mild anomia. Computed tomography (CT) of the brain showed a left hemispheric lesion, without mass effect or contrast enhancement, located in the white matter and sparing the cortex (Fig. 1A). A new brain CT scan was performed after 1 week and revealed an enlargement of the lesion (Fig. 1B). Considering the exposure to rituximab and the clinical picture, on the sixth day from symptoms onset, the cerebrospinal fluid was tested for JCV: a real-time PCR revealed 3,774 copies $/ \mathrm{mL}$.

HIV was negative, helper T cells were 152 cells $/ \mu \mathrm{L}$ (normal range, 410-1,590), and helper $\mathrm{T} /$ cytotoxic $\mathrm{T}$ cells ratio 


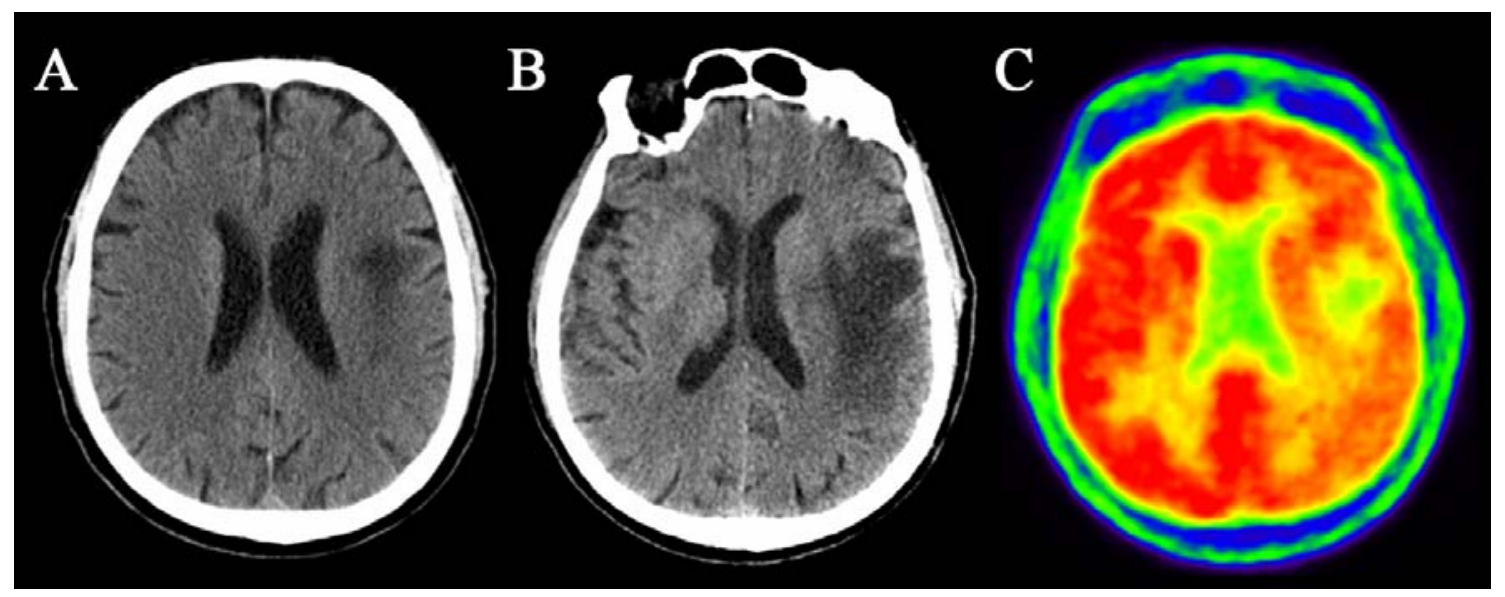

Figure 1. CT and PET scan. (A) CT scan showing subcortical white matter lesion without mass effect and sparing the cortex. (B) Second CT scan showing lesion enlargement. (C) PET scan showing glycemic hypo-metabolism within the lesion.

was 0.84 .

A PET scan showed a glycemic hypo-metabolism in the left hemispheric lesion (Fig. 1C).

On the eleventh day from symptoms onset, the patient was started on mirtazapine (30 mg qd), mefloquine (250 mg qd for 4 days and then $250 \mathrm{mg}$ qw), plasma exchange ( 5 sessions) and, afterwards, intravenous immunoglobulin (30 g daily for 5 days) in order to correct the hypo-gammaglobulinemia due to plasma exchange. On the 25th day, cidofovir (375 mg ow) and interleukin-2 (11 UI qd) were added to the treatment, but the patient continued to deteriorate, developing aphasia at 10 days, right hemiparesis at 3 weeks, stupor at a month, and right ward forced deviation at 5 weeks from symptom onset. In 7 weeks' time, the Glasgow coma scale score was 5, and the patient died after 2 months from the clinical onset.

\section{Discussion}

PML is a rare opportunistic infection showing variable severity, which is affected by many concurrent factors, and among these, the type of immune suppression is highly relevant, as well as its reversibility; in AIDS patients, the introduction of HAART therapy allowed the PML mortality and morbidity to decrease, due to HAART therapy ability to restore the immune-surveillance, thus leading to a better control of JCV opportunistic infection. Different monoclonal antibodies may impair the immune-surveillance with different ways or duration, explaining the different severity and mortality rate observed in natalizumab- and rituximab-associated PML, the former with a $30 \%$ fatality and, sometimes, with a very good recovery [4], the latter with a $90 \%$ fatality [3].

Studies on natalizumab-related PML risk showed a strong effect of serological anti-JCV status, determined by the so-called "stratify" test, a two-step enzyme-linked im- munosorbent assay (ELISA) specifically developed to detect anti-JCV antibodies in human serum or plasma [5].

People who test negative for anti-JCV antibodies have only a low risk to develop PML, which is below 1:10,000 [6], in spite of anti-JCV positive patients which carry a risk around 1:94 if they have been treated for more than 2 years with natalizumab and have an immunosuppressant exposure history.

In the case reported, the patient had both pharmacological (rituximab and CHOP) and clinical (lymphoma) risk factors for PML. In literature, to date there are few data about a potential interaction between these two factors in determining the risk.

In our patient, the diagnosis was made early and multiple therapies (which had previously shown some efficacy in natalizumab-related PML) were administered, but it did not affect the disease course in this case of rituximab-PML.

It should be noted that 3 months had elapsed between last rituximab treatment and PML onset; since a certain amount of antibodies might still be persisting during this period of time, we used plasmapheresis.

A better PML risk stratification based on multiple risk determinants should permit to identify those patients at higher risk and to monitor them clinically and radiologically to detect and treat very early PML; this kind of approach is actually in use for natalizumab-treated patients.

\section{Conclusion}

PML is a rare disorder complicating some monoclonal antibody therapies, with a variable outcome, depending even on the type of immunosuppressant determinants (better for natalizumab, worse for rituximab, with higher mortality). The case may underscore the need for future studies aimed at stratifying PML risk during rituximab therapy, according to several potential factors like JCV serological status, pres- 
ence of multiple immunosuppressant determinants, duration of therapy, and CD4 count.

\section{Grant Support}

This research received no specific grant from any funding agency in the public, commercial, or not-for-profit sectors.

\section{Conflict of Interest}

All the authors declare that they have no conflict of interest regarding the present paper.

\section{Disclosure}

The paper was elaborated at the Specialistic Medicine Department, Neurology Unit, Guglielmo da Saliceto Hospital, Piacenza, Italy, and the corresponding author is Dr Immovilli Paolo.

\section{References}

1. Tavazzi E, Ferrante P, Khalili K. Progressive multifocal leukoencephalopathy: an unexpected complication of modern therapeutic monoclonal antibody therapies. Clin Microbiol Infect. 2011;17(12):1776-1780.

2. Tuccori M, Focosi D, Blandizzi C, Pelosini M, Montagnani S, Maggi F, Pistello M, et al. Inclusion of rituximab in treatment protocols for non-Hodgkin's lymphomas and risk for progressive multifocal leukoencephalopathy. Oncologist. 2010;15(11):1214-1219.

3. Carson KR, Focosi D, Major EO, Petrini M, Richey EA, West DP, Bennett CL. Monoclonal antibody-associated progressive multifocal leucoencephalopathy in patients treated with rituximab, natalizumab, and efalizumab: a Review from the Research on Adverse Drug Events and Reports (RADAR) Project. Lancet Oncol. 2009;10(8):816-824.

4. Vermersch P, Kappos L, Gold R, Foley JF, Olsson T, Cadavid D, Bozic C, et al. Clinical outcomes of natalizumab-associated progressive multifocal leukoencephalopathy. Neurology. 2011;76(20):1697-1704.

5. Gorelik L, Lerner M, Bixler S, Crossman M, Schlain B, Simon K, Pace A, et al. Anti-JC virus antibodies: implications for PML risk stratification. Ann Neurol. 2010;68(3):295-303.

6. Bloomgren G, Richman S, Hotermans C, Subramanyam M, Goelz S, Natarajan A, Lee S, et al. Risk of natalizumab-associated progressive multifocal leukoencephalopathy. N Engl J Med. 2012;366(20):1870-1880. 